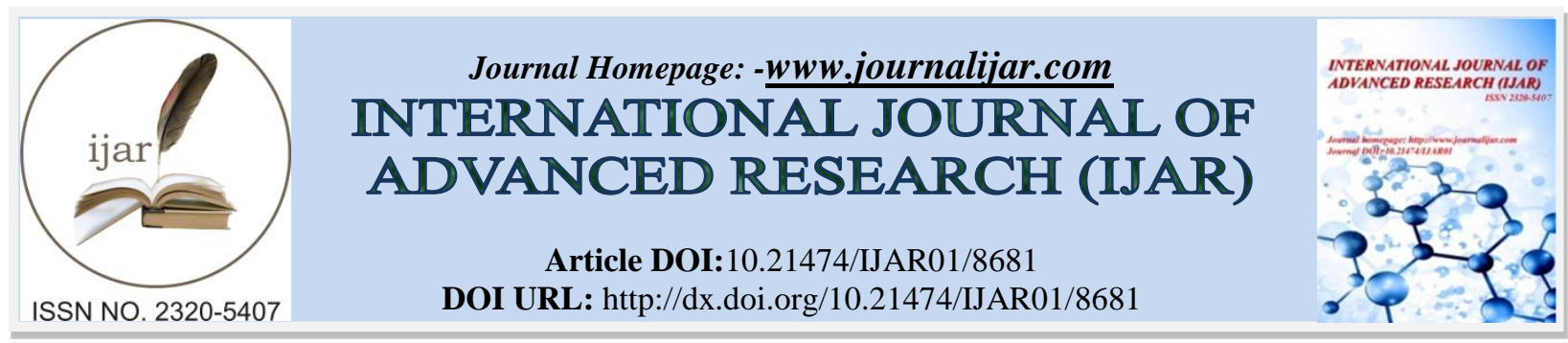

RESEARCH ARTICLE

\title{
KYSTE HYDATIQUE PRIMITIF ET ISOLE DE LA CUISSE: UNE LOCALISATION EXCEPTIONNELLE.
}

Rifki F, Daoudi M, Azriouil O, Mergad O, Nader Y and Koulali Idrissi K.

Service de Traumatologie - Orthopédie, Hôpital Militaire Avicenne, Marrakech, Maroc.

\section{Manuscript Info}

\section{Manuscript History}

Received: 13 January 2019

Final Accepted: 15 February 2019

Published: March 2019

Key words:-

Hydatid disease, cyst, muscle.

\begin{abstract}
Muscular involvement with Echinococcus granulosus is rare, even in endemic countries where the frequency is estimated to be less than $3 \%$, particularly in the countries of the Mediterranean basin, North Africa and Latin America. We report the rare observation of a hydatid cyst of the anterior thigh room in a 26-year-old patient of rural origin with no significant history and was hospitalized in our department for the exploration of a tumefaction Of the antirior surface of the left thigh appeared gradually and insidiously for 6 months. Magnetic resonance imaging (MRI) showed an oval mass evoking an intramuscular hydatid cyst and its relationship to neighboring structures. She underwent surgical excision under locoregional anesthesia. Solitary hydatid localization in muscle tissue is exceptional, but useful to know. Its diagnosis is often delayed, its treatment is surgical, but recurrences are possible.
\end{abstract}

Copy Right, IJAR, 2019,. All rights reserved.

\section{Introduction:-}

L'atteinte musculaire par l'Echinococcus granulosus est rare, même dans les pays endémiques où sa fréquence est estimée à moins de $3 \%$ en particulier dans les pays du bassin méditerranéen, d'Afrique du Nord et d'Amérique latine [1]. La localisation musculaire primitive et isolée d'un kyste hydatique (KH) est exceptionnelle [2]. La plupart des kystes $(60 \%)$ se développent dans le foie; les autres sites de développement sont le poumon (20\%) et moins fréquemment la rate, le cœur, le rein, le système nerveux central, et l'os [3]. Nous rapportons l'observation rare d'un kyste hydatique isole de la loge antérieure de la cuisse gauche chez une patiente âgée de 26 ans habitant le milieu rural et nous discutons les aspects épidémiologiques, les difficultés diagnostiques et les modalités thérapeutiques de cette pathologie

\section{Observation:-}

Il s'agit d'une patiente âgée de 26 ans, d'origine rurale, n'ayant aucun antécédent notable, avait été hospitalisé dans notre service pour exploration d'une tuméfaction de la face antérieure de la cuisse gauche apparue de façon progressive et insidieuse depuis 6 mois sans notion de douleur du membre inferieure gauche ou de signes inflammatoires.

À l'examen, elle était apyrétique et en bon état général. La palpation de la face antérieure de la cuisse gauche retrouvait une masse volumineuse, mal limitée, mobile par rapport aux plans superficiels et profonds, légèrement sensible avec une peau en regard normale et sans signes de compression nerveuse ou vasculaire. 
Les radiographies standard avaient objectivé un épaississement des tissus mous sans calcifications et sans anomalie osseuse. La radiographie thoracique était normale.

L'IRM de la cuisse montrait une masse ovalaire kystique multiloculaire mesurant $8 \mathrm{~cm}$ de longueur sur $5 \mathrm{~cm}$ de large et 4,5cm d'épaisseur, contenant des cloisons avec de petites image arrondies de signal liquidien évoquant un kyste hydatique intramusculaire du vaste intermédiaire stade III (figure 1) .le bilan biologique avait montre une hyperéosinophilie à 5,1\%, avec une sérologie hydatique positive. Le scanner thoraco-abdomino-pelvien ne révélait pas d'autres localisations.

Elle a bénéficié d'une exérèse chirurgicale sous rachianesthésie (figure2). En peropératoire, le plan de clivage était non évident, et accolé a 1 artère fémorale superficielle. Malgré cela, nous réussîmes à réaliser une périkystectomie (figure3) emportant la tumeur en totalité. L'ouverture de la pièce opératoire a trouvé de multiples vésicules filles (figure 4). L'étude parasitologique a montré de nombreux protoscolexes d'Echinococcus granulosus (figure 5). Les suites opératoires ont été simples. Un traitement médical a été suivi pendant 6 mois. Après un recul d'une année, la surveillance clinique, sérologique et échographique n'a pas objectivé de récidive.

\section{Discussion:-}

L'hydatidose est une maladie endémique qui pose un problème de santé publique dans certaines régions du monde où s'effectue l'élevage du bétail et des moutons, notamment dans le pourtour méditerranéen, le Moyen-Orient et l'Amérique du Sud [4].Cette parasitose est causée par la larve de Taenia Echinococcus granulosus qui vit dans le tube digestif des chiens, domestiques ou sauvages, et d'autres animaux carnivores dont ils sont les hôtes définitifs. Les humains en sont les hôtes intermédiaires accidentels lorsqu'ils ingèrent des oeufs de Taenia [5].

Le foie (60\%des cas) et le poumon (20\% des cas) représentent les deux localisations les plus fréquentes. Ceci peut être expliqué par le rôle physiologique des organes. Ainsi ils constituent un double filtre physiologique à la dissémination du parasite, rendant les localisations extra-hépato-pulmonaires moins fréquentes [6]. La période d'incubation peut durer de cinq à 20 ans et les symptômes surviennent plusieurs années après l'exposition [7].

Le kyste hydatique primitif musculaire est rare même en zones endémiques. Sa fréquence varie de 1 à $5 \%$. Les embryons hexacanthes qui pénètrent par voie digestive sont le plus souvent arrêtés par les filtres que constituent le foie et les poumons [3]. L'atteinte musculaire primitive est décrite dans la littérature pour la musculature de la paroi thoracique, le grand pectoral, le sartorius, le quadriceps et le glutéus $[6,7,8]$.

Dans le cas présenté, la localisation musculaire se situait au niveau de la loge antérieure de la cuisse. L'IRM est l'examen de choix en pathologie hydatique des parties molles [5]. Elle permet, grâce à sa haute résolution en contraste, de mieux étudier l'extension locorégionale de la lésion et ses rapports avec les pédicules vasculo-nerveux, tout en offrant une analyse minutieuse des parois kystiques.

Les biopsies à l'aiguille guidées par les ultrasons sont aussi une aide au diagnostic, bien que certains auteurs ne le recommandent pas du fait du risque de rupture kystique et de réaction anaphylactique [9]. Le résultat des analyses microscopiques de la biopsie à l'aiguille n'est pas garanti, surtout si les patients ont reçu un traitement médical, et la région de la biopsie doit être réséquée dans le temps de la chirurgie [4]. Divers tests sérologiques peuvent aider au diagnostic d'échinococcose. Cependant, la sérologie seule n'est pas suffisante pour le diagnostic. On retrouve 50\% de faux négatif chez les patients porteurs d'un kyste pulmonaire solitaire [10]. La sensibilité et la spécificité des tests sérologiques ne sont pas connues chez les patients atteints d'échinococcose des tissus mous. Cette sérologie peut être utile pour la surveillance du traitement lorsqu'elle est positive [4]

Le traitement de l'échinococcose musculaire est chirurgical [4]. La chirurgie doit être prudente évitant ainsi l'ouverture du kyste pendant la dissection. Il faut protéger le champ opératoire par une solution de sérum salé hypertonique et/ou d'eau oxygénée dès l'abord chirurgical [11]. La technique de choix est la périkystectomie, emportant le kyste en entier sans effraction de sa paroi. L'exérèse en monobloc avec périkystectomie totale est le procédé idéal, mais pas toujours réalisable surtout si le kyste est volumineux, profond prenant contact avec les éléments vasculo-nerveux avoisinants [11].

L'intérêt du traitement médical par les dérivés de benzimidazole (albendazole) dans les localisations solitaires de l'appareil locomoteur reste discuté en raison de leurs mauvaise diffusion dans le liquide kystique [4, 12]. Ce 
traitement est réservé aux cas inopérables ou en complément de la chirurgie lorsque le kyste s'est compliqué de rupture [13.14].

\section{Conclusion:-}

La localisation hydatique solitaire dans les tissus musculaires est exceptionnelle, mais utile à connaître. Le tableau clinique est souvent pauvre et peut mimer une tumeur des tissus mous. Le diagnostic est confirmé par l'IRM. Le traitement est chirurgical.

\section{Conflit d'intérêt}

Pas de conflits d'intérêt.

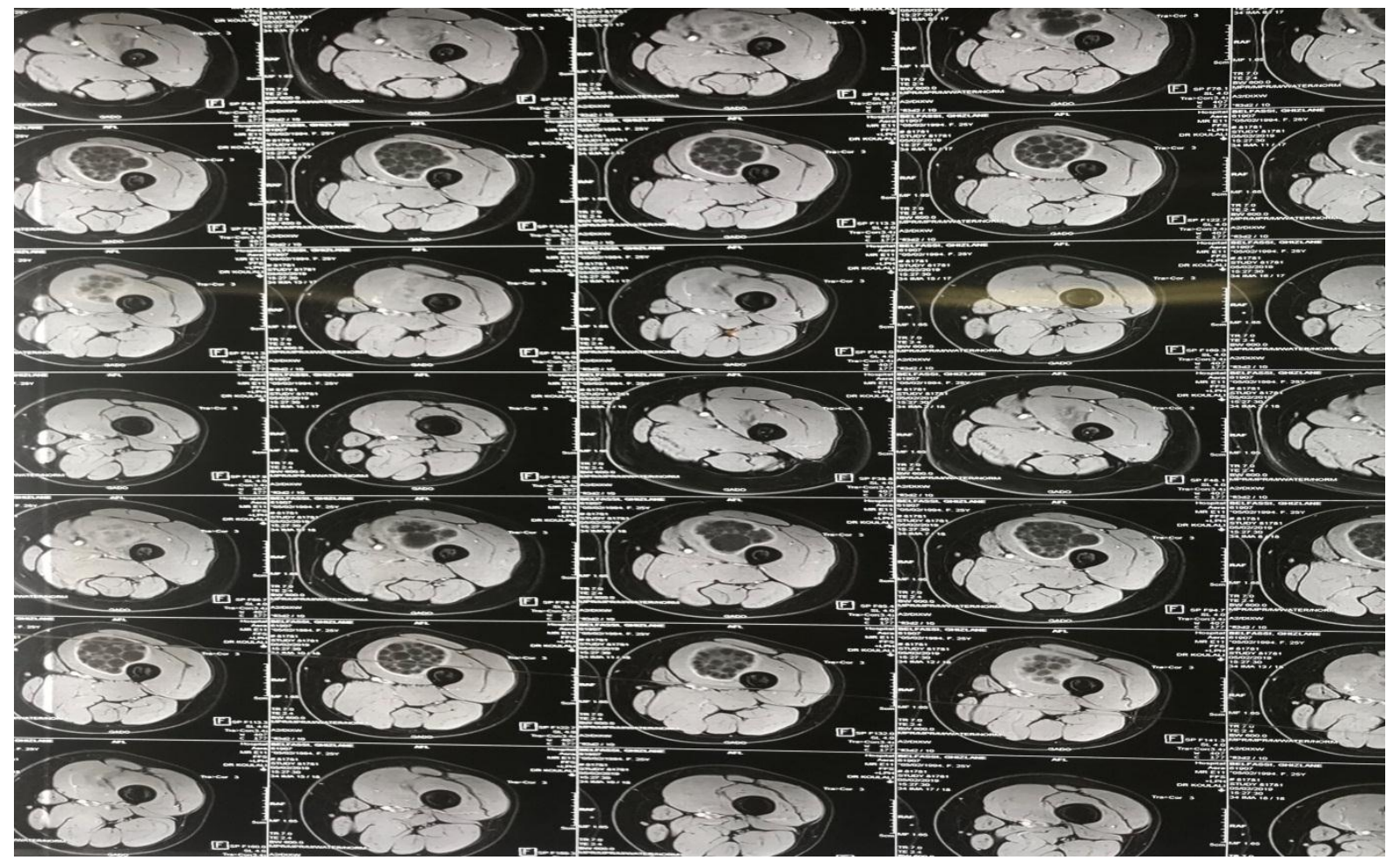

Figure 1:-Aspect en imagerie par résonance magnétique (IRM) du kyste hydatique $(\mathrm{KH})$ intramusculaire.

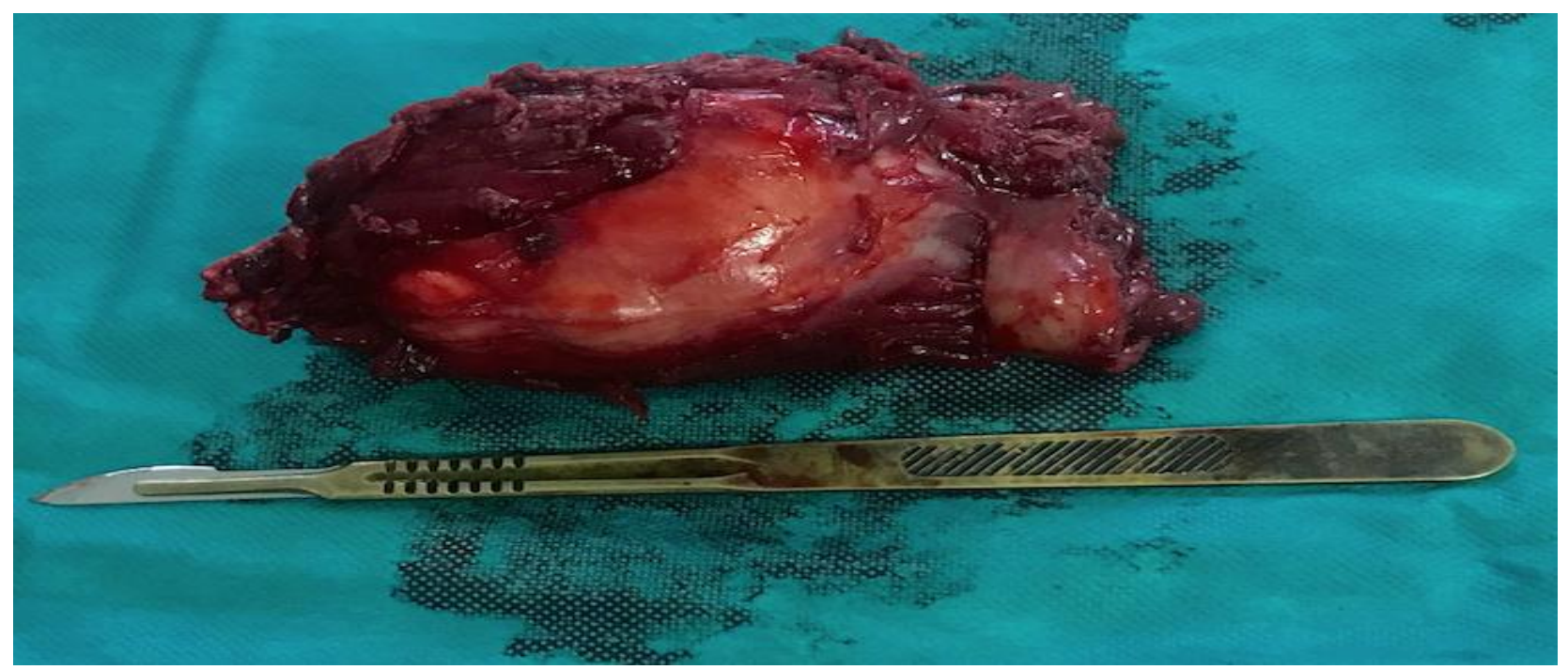

Figure 2:-Pièce d'exérèse 


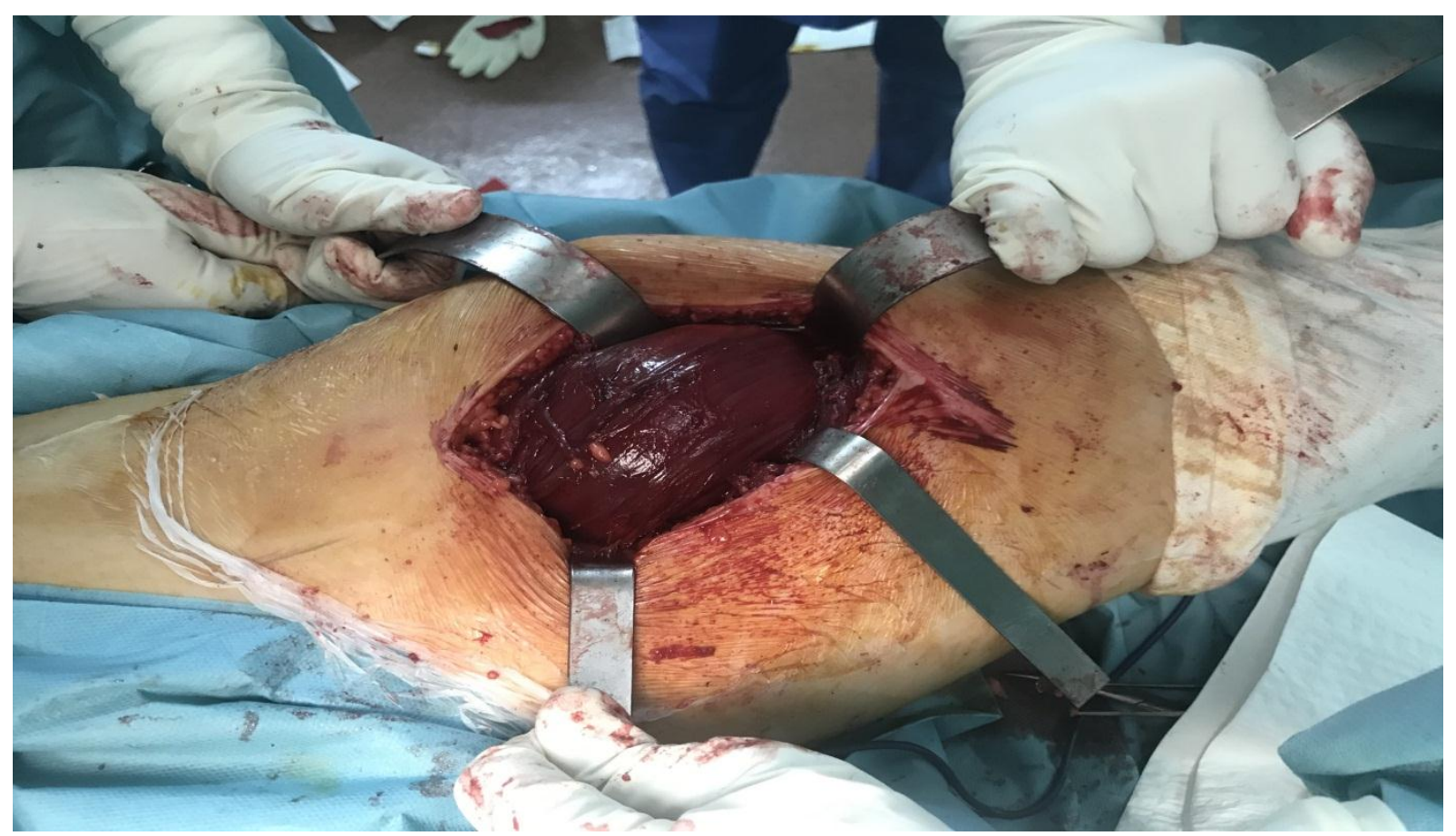

Figure 3:-Perikystectomie de la tumeur

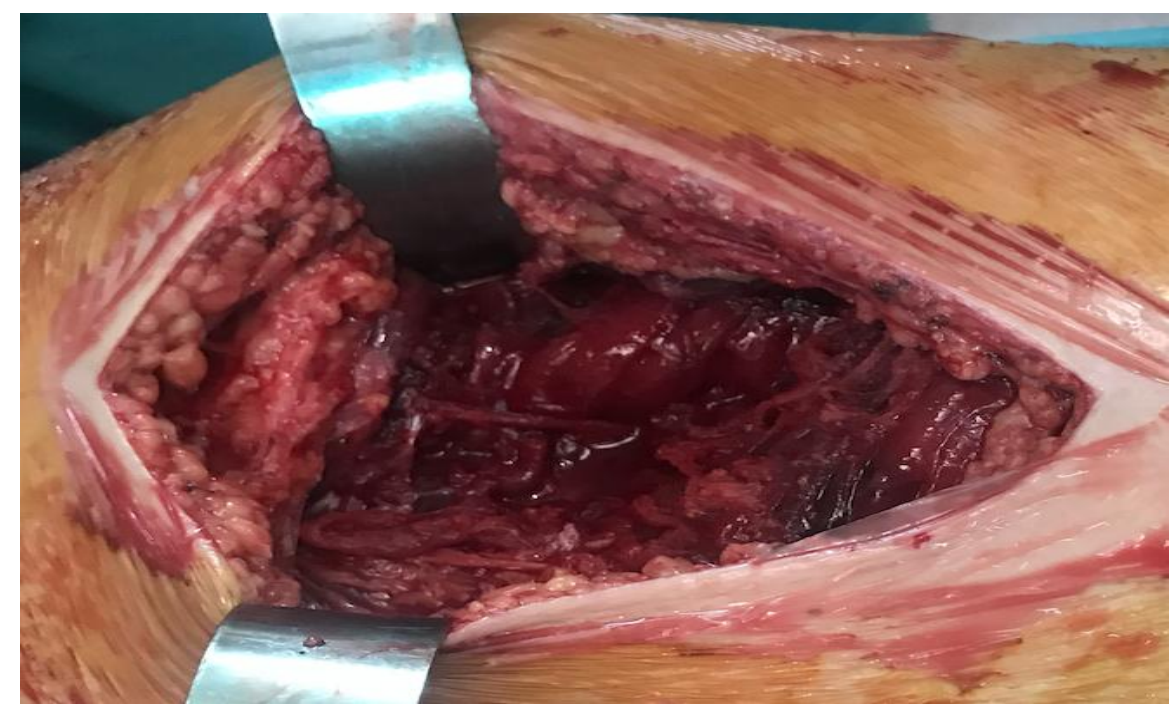

Figure 4:-après périkystectomie 


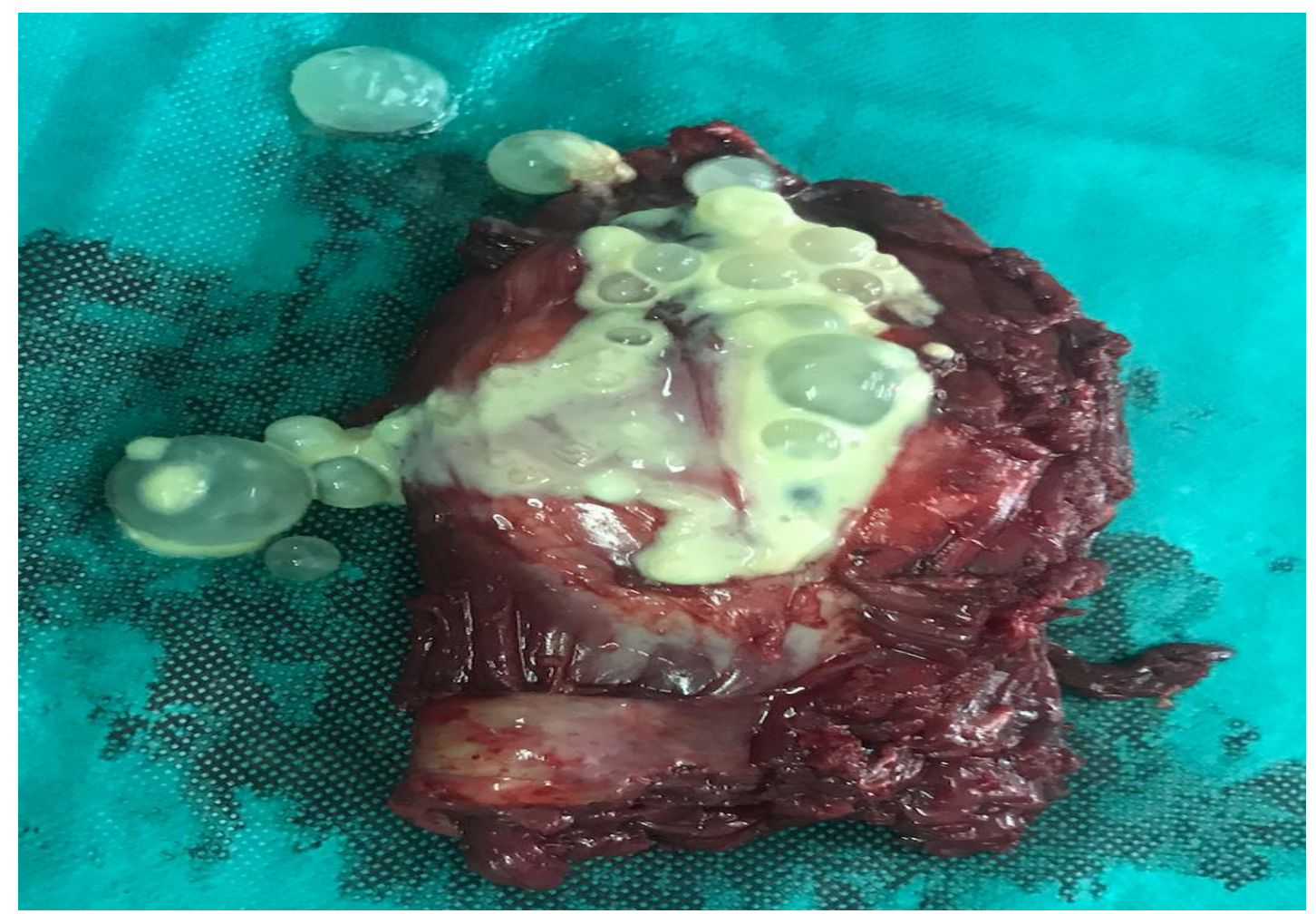

Figure 4:-Ouverture de la pièce opératoire et récupération de vésicules filles

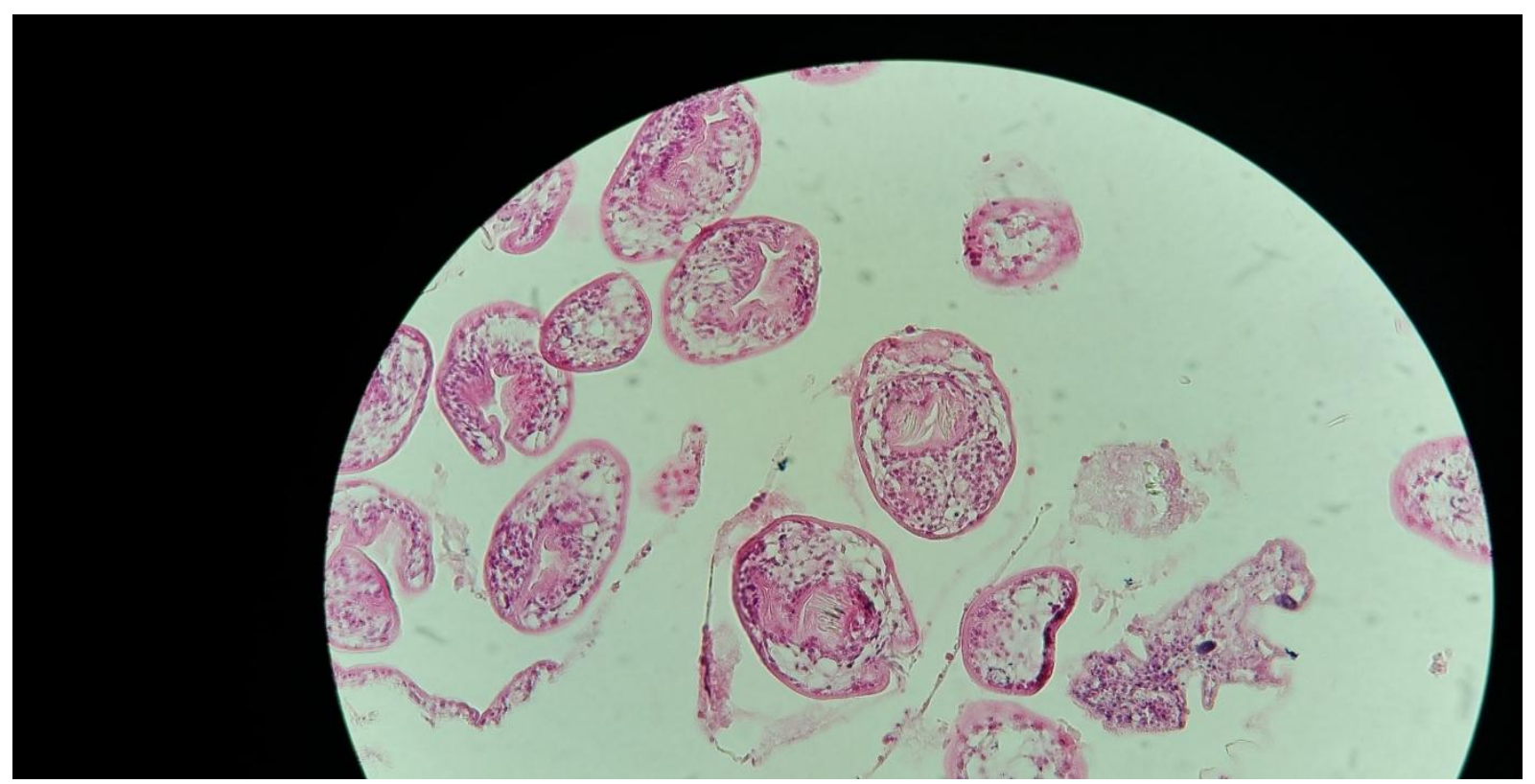

Figure 5:-Protoscolex au fort grossissement 


\section{Référence:-}

1. Kazakos CJ, Galanis VG, Verettas DA, Polychronidis A, Simopoulos C. Primary hydatid disease in femoral muscles. J Int Med Res 2005;33:703 - 6.

2. Cissé AM, Nassar I, Hammani L, Dafiri R, Imani F. Hydatidose primitive

3. et étendue de la cuisse : aspect radiologique inhabituel.J Radiol 2002;83:1778—80.

4. S. Jerbi Omezzine et al. Kyste hydatique primitif de la cuisse. Une localisation rare. Revue de chirurgie orthopédique et traumatologique (2010) 96, 105-108

5. M.F. Amar et al. Journal de Traumatologie du Sport 27 (2010) 146-148

6. K. Rafiqi, A. Rafaoui , M. Sirrajelhak, A. Messoudi, H.A. Abouali ,M. Rafai, M. Rahmi, A. Garch. Kyste hydatique primitif de la cuisse chez un bodybuilder. À propos d'un cas et revue de la littérature. Journal de Traumatologie du Sport 33 (2016) 107-109

7. Dawson JL, Stamatakis JD, Stringer MD, Williams R. Surgical treatment of hepatic hydatid disease. Br J Surg 1988;75:946-50.

8. Ozkoc G, Akpinar S, Hersekli MA, Ozalay M, Tandogan R. Primaryhydatiddisease of the quadriceps muscle: a rare localization. Arch Orthop TraumaSurg 2003;123:314-6.

9. Combalia A, Sastre S. Kyste hydatique du muscle glutéal. Deux cas. Revuede la littérature. Rev Rhum 2005;72:851-7.

10. Ozkoc G, Akpinar S, HersekliMA,OzalayM,Tandogan R. Primary hydatid disease of the quadriceps muscle: a rare localization. Arch Orthop Trauma Surg 2003;123:314-6.

11. Kammerer WS, Schantz PM. Echinococcal disease. Infect Dis Clin North Am 1993;7:605-18.

12. M. Mseddi, M. Mtaoumi, J. Dahmene, R. Ben Hamida, A. Siala, T. Moula, M.L. Ben AyecheHydatid cysts in muscles: eleven cases. Revue de chirurgie orthopédique. 2005, 91, 267-271.

13. Hammami T, Noomane F, Ketata M, Ganneme Y, Nasr M, Zouari K, et al.Kyste hydatique musculaire de la cuisse. À propos de trois observations.Rev Chir Orthop Reparatrice Appar Mot 2002;88:193-6.

14. Daoudi A, Loudiyi WD, Elibrahimi A, Elmrini A, Chakour K, Bou-tayeb F. Le kyste hydatique sous-cutané solitaire de la région glutéale :une localisation inhabituelle. À propos d'un cas. Ann Chir Plast Esthet2008;53:448-51.

15. Daoudi A, Loudiyi WD, Elibrahimi A, Elmrini A, Chakour K, Boutayeb F. Le kyste hydatique sous-cutané solitaire de la région glutéale : une localisation inhabituelle. À propos d'un cas. Ann Chir Plast Esthet 2008;53:448-51. 